\title{
Thrombectomy Outcomes in Acute Ischemic Stroke due to Middle Cerebral Artery M2 Occlusion with Stent Retriever versus Aspiration: A Multicenter Experience
}

\author{
Kunakorn Atchaneeyasakula ${ }^{a} \quad$ Amer M. Malik $^{a}$ Dileep R. Yavagala, b \\ Diogo C. Haussen ${ }^{c}$ Ashutosh P. Jadhav ${ }^{d}$ Mehdi Bouslama ${ }^{c}$

 \\ Seemant Chaturvedi ${ }^{a}$ Tudor G. Jovin ${ }^{d}$ Raul G. Nogueira ${ }^{c}$ \\ a Department of Neurology, University of Miami Miller School of Medicine, Miami, FL, USA; \\ ${ }^{b}$ Department of Neurosurgery, University of Miami Miller School of Medicine, Miami, FL, \\ USA; ' ${ }^{C}$ Department of Neurology, Emory University School of Medicine, Atlanta, GA, USA;

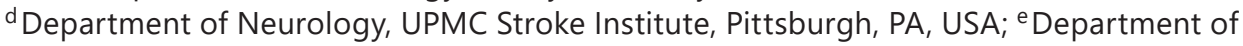 \\ Neurosurgery, Emory University School of Medicine, Atlanta, GA, USA
}

\section{Keywords}

Acute ischemic stroke - Thrombectomy - Endovascular therapy - Middle cerebral artery occlusion $\cdot \mathrm{M} 2$

\begin{abstract}
Objective: To examine outcomes for thrombectomy devices used for treatment of acute ischemic stroke (AIS) with middle cerebral artery (MCA) M2 segment emergent large vessel occlusion (ELVO) as the optimal device for such reperfusion is not clearly defined. Methods: A retrospective cohort study of consecutive AIS patients with MCA M2 ELVO undergoing thrombectomy from 3 academic medical centers was conducted from October 1999 through June 2016. The patients were divided based on the device utilized. Multivariate analysis of associations between devices (stent retriever or aspiration only [manual or pump aspiration system]) was performed. Primary outcomes were good recanalization (i.e., modified thrombolysis in cerebral infarction score $\geq 2 \mathrm{~b}$ ) and a favorable modified Rankin scale (mRS) score (i.e. $\leq 2)$. The secondary outcome was symptomatic intracerebral hemorrhage (sICH). Results: A total of 197 AIS patients underwent MCA M2 ELVO thrombectomy with either a stent retriever $(n=120)$ or aspiration only $(n=77)$. The aspiration-only group utilized either manual $(n=38)$ or pump aspiration ( $n=39$ ). Utilization of a stent retriever over manual aspiration is independently associated with higher odds of a favorable $\mathrm{mRS}$ score $(\mathrm{OR}=3.2 ; 95 \% \mathrm{Cl} 1.02-9.7)$ and lower odds of $\mathrm{sICH}(\mathrm{OR}=0.09 ; 95 \% \mathrm{Cl} 0.03-0.31)$. Utilization of a stent retriever over a pump aspiration
\end{abstract}

K. Atchaneeyasakul and A.M. Malik contributed equally to this work and are co-first-authors. 
system is independently associated with higher odds of good recanalization (OR $=3.8 ; 95 \%$ $\mathrm{Cl}$ 1.5-9.6). Utilization of a newer-generation pump aspiration catheter compared to a stent retriever resulted in similar rates of favorable mRS scores, $\mathrm{slCH}$, successful recanalization, and mortality. Conclusion: Utilization of a newer-generation pump aspiration catheter compared to a stent retriever resulted in similar outcomes, but worse outcomes were seen with the manual aspiration technique. These findings need to be confirmed with a large randomized trial utilizing stent retrievers and newer-generation pump aspiration systems.

(c) 2019 S. Karger AG, Basel

\section{Introduction}

Recent randomized endovascular stroke treatment trials have demonstrated that mechanical thrombectomy with a stent retriever can improve functional outcomes in anterior circulation acute ischemic stroke (AIS) due to emergent large vessel occlusion (ELVO) of the intracranial internal carotid artery (ICA) and the middle cerebral artery (MCA) M1 segment [1]. The most recent American Heart Association guidelines from 2015 regarding endovascular AIS treatment recommend the use of stent retriever devices for ELVO thrombectomy of the M1 segment within $6 \mathrm{~h}$ of stroke onset [2]. However, such data regarding AIS due to MCA M2 segment ELVO are limited. Post hoc analysis of the STAR, SWIFT, and SWIFT-PRIME trials found thrombectomy in MCA M2 occlusion to be feasible in achieving successful reperfusion [3]. A recently published multicenter retrospective study found that thrombectomy in ELVO of the M2 segment led to a high probability of successful reperfusion which resulted in significantly improved rates of good outcomes as compared to medical therapy [4]. At present, neurointerventionalists utilize multiple techniques for ELVO thrombectomy of the M2 segment, especially stent retrievers or aspiration either manually or with a pump aspiration device. However, the most optimal technique and/or device for such reperfusion is not clearly defined. We aim to compare the outcomes for the techniques and devices used for thrombectomy of AIS patients due to MCA M2 ELVO.

\section{Methods}

This analysis is a retrospective cohort study of consecutive AIS patients with anterior circulation ELVO undergoing thrombectomy from 3 large volume tertiary care academic medical centers. This study received approval from the institutional review boards of each institution. The inclusion criteria include patients with ELVO of the MCA M2 segment without the involvement of other segments proximal to the occlusion site as confirmed by cerebral catheter angiography.

Patient demographics, including age at diagnosis, gender, medical comorbidities (hypertension, diabetes mellitus, hyperlipidemia, coronary artery disease, and atrial fibrillation), and smoking history were obtained from the prospectively collected institutional stroke databases. Baseline National Institute of Health Stroke Scale (NIHSS) scores were obtained from the same data sources. We also recorded the last known well-togroin puncture time as well as the type of thrombectomy device utilized.

The outcomes measured were: successful angiographic reperfusion, defined by a modified thrombolysis in cerebral infarction (mTICI) score $\geq 2 \mathrm{~b}$; the rate of symptomatic intracerebral hemorrhage (sICH), defined by parenchymal hematoma with worsening NIHSS score ( $\geq 4$ points) as per the ECASS-II study definition [5]; a favorable modified Rankin scale (mRS) score $\leq 2$ at 90 days from stroke onset; and mortality. In our study, the mRS score at 90 days recorded in the database was derived from patient follow-up visit documentation closest to 90 days after stroke onset or a telephone follow-up visit.

Outcomes were compared between the stent retriever group and the aspiration-only group. The stent retriever group consisted of patients who received treatment with a stent retriever alone or in combination with aspiration devices. The aspiration-only group consisted of patients who received treatment with either 
a pump aspiration system or manual aspiration. The manual aspiration technique utilized was described by Jankowitz et al. [6]. Subgroup analysis of stent retriever versus pump aspiration and manual aspiration was also performed. In addition, analysis of stent retriever versus newer-generation pump aspiration devices, including the Penumbra system ACE or MAX series (Penumbra, Alameda, CA, USA) was performed.

Statistical analysis was performed using the Statistical Package for the Social Sciences (SPSS, version 24; IBM Corporation, Armonk, NY, USA). Student's $t$ test, The Mann-Whitney test, and the $\mathrm{X}^{2}$ test were used as appropriate for univariate analysis. Multivariate logistic regression analyses were performed to evaluate the predictors of each primary outcome and safety end points. Variables with $p<0.1$ from the univariate logistic regression analysis were included in the multivariate logistic regression analysis. $p<0.05$ was considered statistically significant.

\section{Results}

From October 1999 through June 2016, a total of 4,478 AIS patients underwent mechanical thrombectomy; 272 AIS patients underwent thrombectomy for MCA M2 ELVO at our 3 academic medical centers, and 197 AIS patients underwent thrombectomy with a stent retriever $(n=120)$ or aspiration only (manual or pump aspiration system) $(n=77)$ and were included in this study. The aspiration-only group utilized either manual aspiration $(n=38)$ or a pump aspiration system $(n=39)$. The catheters utilized with manual aspiration include Penumbra 4MAX (Penumbra) in $31.6 \%$ of the cases, Penumbra 5MAX (Penumbra) in $2.6 \%$ of the cases, DAC (Concentric Medical, Mountain View, CA, USA) in $21.1 \%$ of the cases, and Navien (Covidien Vascular Therapies, Mansfield, MA, USA) in 28.9\% of the cases. The type of device was not documented in $15.8 \%$ of the cases. Table 1 shows the baseline characteristics. There was no significant difference in baseline characteristics between the stent retriever group and the aspiration group.

The outcome univariate analysis of patients with M2 vessel occlusion in the stent retriever group compared to the aspiration group is presented in Table 2. Between the stent-retriever group and the aspiration group (both the manual and the pump aspiration groups), there was a significant difference in the rate of good recanalization (90 vs. 77.3\%; $p<0.016$ ) and the rate of sICH (3.4 vs. $16.7 \% ; p=0.001$ ). There was no significant difference in favorable $\mathrm{mRS}$ between the stent retriever group and the aspiration group ( $52.1 \mathrm{vs.} 36.7 \%, p=0.06)$. In the analysis by aspiration technique, between the stent retriever group and the manual aspiration group there was a significant difference in the rate of favorable mRS (52.1 vs. 26.1\%; $p=0.025)$, the rate of sICH (3.4 vs. $26.1 \%$; $p<0.001$ ), and the mortality rate ( 9.3 vs. $31.6 \%$; $p=0.001$ ). Between the stent retriever group and the pump aspiration system group, there was a significant difference in the rate of good recanalization (90 vs. 70.3\%; $p<0.003$ ) but there was no significant difference in the rate of favorable mRS (52.1 vs. $43.2 \% ; p=0.361$ ).

The multivariate analysis for predictors of outcomes in mechanical thrombectomy for M2 vessel occlusion is shown in Table 3. Utilization of a stent retriever over manual aspiration was independently associated with higher odds of a favorable mRS score (OR $=3.17 ; 95 \%$ CI 1.03-9.75) and lower odds of sICH (OR $=0.09$; 95\% CI 0.026-0.311) and mortality (OR = 0.228 ; $95 \%$ CI $0.085-0.613$ ). Utilization of a stent retriever over a pump aspiration system was independently associated with higher odds of good recanalization (OR $=3.81$; $95 \% \mathrm{CI}$ 1.51-9.59). Age is also an independent predictor of a higher sICH rate, a higher mortality rate, and a lower rate of favorable $\mathrm{mRS}$.

A separate analysis of outcomes by univariate and multivariate analysis of patients with M2 vessel occlusion in the stent retriever group compared to the newer-generation pump aspiration catheter group is presented in Table 4. Utilization of a newer-generation pump aspiration catheter compared to a stent retriever resulted in similar rates of favorable mRS, sICH, successful recanalization, and mortality. 
Table 1. Baseline characteristics of patients with $M 2$ vessel occlusion in the stent retriever group compared to the aspiration group

\begin{tabular}{lccc}
\hline & $\begin{array}{l}\text { Stent retriever } \\
(n=120)\end{array}$ & $\begin{array}{l}\text { Aspiration } \\
(n=77)\end{array}$ & $p$ value \\
\hline Females & 40 & 42.9 & 0.691 \\
Age, years & $68.6 \pm 13.1$ & $67.7 \pm 13.4$ & 0.653 \\
Diabetes mellitus & 31.1 & 30.3 & 0.903 \\
Hypertension & 72.3 & 77.6 & 0.403 \\
Hyperlipidemia & 33.6 & 43.7 & 0.166 \\
Atrial fibrillation & 40.3 & 28.9 & 0.106 \\
Last known well-to-groin puncture & $280(209-443)$ & $276.5(173.75-425)$ & 0.399 \\
$\quad$ time, min & $15(11-20)$ & $17(12-20)$ & 0.134 \\
Initial NIHSS score & 55.2 & 36.4 & 0.098 \\
IV-tPA & $9(7.5-9.5)$ & $9(7-10)$ & 0.809 \\
ASPECTS & & &
\end{tabular}

Values are presented as means $\pm S D$, medians (IQR), or percents.

Table 2. Univariate analysis of the outcomes of patients with $M 2$ vessel occlusion in the stent retriever group compared to the aspiration group

\begin{tabular}{|c|c|c|c|c|c|c|c|}
\hline \multirow{3}{*}{$\begin{array}{l}\text { Good recanalization } \\
\text { sICH }\end{array}$} & \multicolumn{2}{|c|}{$\begin{array}{l}\text { Stent retriever Aspiration }{ }^{\text {a }} \\
(n=120) \quad(n=77)\end{array}$} & \multirow{2}{*}{$\begin{array}{c}p \text { value } \\
0.016^{*}\end{array}$} & \multirow{2}{*}{$\begin{array}{l}\text { Manual } \\
\text { aspiration } \\
(n=38)\end{array}$} & \multirow{2}{*}{$\begin{array}{c}p \text { value } \\
0.346\end{array}$} & \multicolumn{2}{|c|}{$\begin{array}{l}\text { Pump aspiration } p \text { value } \\
\text { system } \\
(n=39)\end{array}$} \\
\hline & 90 & 77.3 & & & & 70.3 & $0.003^{*}$ \\
\hline & 3.4 & 16.7 & $0.001^{*}$ & 28.6 & $<0.001^{*}$ & 5.4 & 0.572 \\
\hline Favorable mRS score & 52.1 & 36.7 & 0.06 & 26.1 & $0.025^{*}$ & 43.2 & 0.361 \\
\hline Death & 9.3 & 21.1 & $0.025^{*}$ & 31.6 & $0.001^{*}$ & 10.5 & 0.832 \\
\hline 90-day mRS score & $2(1-4)$ & $4(2-6)$ & $0.02^{*}$ & $6(2-6)$ & $0.005^{*}$ & $3(1-5)$ & 0.268 \\
\hline
\end{tabular}

Values are presented as medians (range) or percents. ${ }^{a}$ Dichotomized into manual aspiration and pump aspiration system. *Asterisks denote statistically significant values in relation to the specified $p$ value in Methods.

\section{Discussion}

Current evidence from recent randomized trials supports the utilization of a stent retriever for thrombectomy of anterior circulation ELVO, including ICA and M1 segment of MCA [1]. The efficacy of mechanical thrombectomy of the M2 portion of MCA ELVO is understudied and the optimal technique and/or device is not clearly defined. In this study we found that utilization of a stent retriever over a pump aspiration system is independently associated with a higher recanalization rate with no significant difference in favorable outcomes. However, in a subgroup analysis of newer-generation pump aspiration catheter versus stent retriever, there was no significant difference in outcomes, including rates of favorable mRS, successful recanalization, sICH, and mortality.

At present, the available data on thrombectomy of M2 ELVO from the pooled meta-analysis of randomized control thrombectomy trials do not have enough patients to show a significant improvement in outcomes [1]. However, a multicenter retrospective trial was performed that included a larger number of M2 ELVO patients, with 288 patients in the mechanical thrombectomy group and 234 patients in the medical management group. That study found that 
Table 3. Multivariate logistic regression analysis of predictors of outcomes in mechanical thrombectomy for M2 vessel occlusion

\begin{tabular}{lcc}
\hline Variable & OR $(95 \% \mathrm{CI})$ & $p$ value \\
\hline Predictors of good recanalization & & $0.018^{*}$ \\
Stent retriever vs. aspiration & $2.638(1.179-5.9)$ & 0.332 \\
Stent retriever vs. manual aspiration & $1.688(0.587-4.853)$ & $0.005^{*}$ \\
Stent retriever vs. pump aspiration system & $3.808(1.512-9.587)$ & \\
\hline Predictors of sICH & & $0.044^{*}$ \\
Age & $1.074(1.002-1.151)$ & $0.003^{*}$ \\
Stent retriever vs. aspiration & $0.17(0.052-0.553)$ & $<0.001^{*}$ \\
Stent retriever vs. manual aspiration & $0.09(0.026-0.311)$ & 0.383 \\
Stent retriever vs. pump aspiration system & $0.447(0.073-2.728)$ & $<0.0001^{*}$ \\
\hline Predictors of a favorable mRS score & & 0.337 \\
Age & $0.922(0.891-0.955)$ & $0.023^{*}$ \\
Hypertension & $0.857(0.295-1.519)$ & $0.034^{*}$ \\
Initial NIHSS score & $0.926(0.867-0.989)$ & $0.045^{*}$ \\
Stent retriever vs. aspiration & $2.267(1.064-4.829)$ & 0.148 \\
Stent retriever vs. manual aspiration & $3.165(1.028-9.747)$ & $0.001^{*}$ \\
Stent retriever vs. pump aspiration system & $1.916(0.794-4.625)$ & 0.91 \\
\hline Predictors of mortality & & 0.283 \\
Age & $1.086(1.034-1.141)$ & $0.033^{*}$ \\
Atrial fibrillation & $0.947(0.366-2.451)$ & $0.003^{*}$ \\
Initial NIHSS score & $1.05(0.961-1.147)$ & 0.892 \\
Stent retriever vs. aspiration & $0.374(0.151-0.923)$ & $0.228(0.085-0.613)$ \\
Stent retriever vs. manual aspiration & $0.908(0.223-3.693)$ & \\
\hline
\end{tabular}

Variables with $p<0.1$ in the univariate analysis were included in the analysis. *Asterisks denote statistically significant values in relation to the specified $p$ value in Methods.

Table 4. Univariate and multivariate analysis of the outcomes of patients with $\mathrm{M} 2$ vessel occlusion in the stent retriever group compared to the newer-generation pump aspiration catheter group

\begin{tabular}{lclll}
\hline Outcome & $\begin{array}{l}\text { Stent retriever } \\
(n=120)\end{array}$ & $\begin{array}{l}\text { Newer-pump } \\
\text { aspiration catheter } \\
(n=22)\end{array}$ & $\begin{array}{l}\text { Univariate } \\
\text { analysis } \\
p \text { value }\end{array}$ & $\begin{array}{l}\text { Multivariate } \\
\text { analysis } \\
p \text { value }\end{array}$ \\
\hline Good recanalization & 90.0 & 86.4 & 0.61 & 0.59 \\
sICH & 3.4 & 4.8 & 0.75 & 0.92 \\
Favorable mRS score & 52.1 & 52.4 & 0.98 & 0.38 \\
Death & 9.3 & 9.5 & 0.98 & 0.23 \\
\hline
\end{tabular}

Values are presented as percents.

mechanical thrombectomy in ELVO of the M2 segment significantly improved the rate of good outcomes [4]. A single-institution study comparing pump aspiration thrombectomy and stentretriever thrombectomy did not show a significant difference in clinical outcomes or successful recanalization rates, which could be due to underpowering as a result of a small sample size [7]. With the aim of increasing the power, our collaborative from 3 tertiary care academic centers was able to show that utilization of a stent retriever over manual aspiration is independently associated with higher odds of a favorable mRS score. Utilization of a stent retriever 
over a pump aspiration system is associated with higher odds of a good recanalization rate but we were unable to detect a significant difference in favorable outcomes (52.1 vs. $43.2 \%)$. As recently reported in the ASTER randomized clinical trial evaluating the efficacy of contact aspiration versus stent retriever in AIS and large vessel occlusion not limited to M2 ELVO, this study found the recanalization rate to be $83.1 \%$ in the stent retriever group and $85.4 \%$ in the contact aspiration group [8]. Even though in the ASTER trial M2 ELVO was not the only target lesion, the lower recanalization rate of pump aspiration system thrombectomy in our cohort compared to the ASTER trial may be due to our study including patients dating back to 1999 and utilization of an older-generation aspiration system. In our subgroup analysis of stent retriever versus newer-generation pump aspiration catheter, including the Penumbra system ACE or MAX series similar to the ASTER trial, we found similar rates of favorable mRS, sICH, successful recanalization, and mortality. The better outcome may be related to the newergeneration pump aspiration catheters designed to have better tracking technology and maneuverability and a larger catheter lumen diameter. High successful recanalization rates were seen with newer-generation pump aspiration catheters (86.4\%) and stent retrievers (90\%). Regarding the sICH rate in our cohort, in the stent retriever group it was less (i.e., 3.4\%) than in the manual aspiration group (i.e., 28.6\%), but it was similar to the rate in the pump aspiration group (i.e., 5.4\%). The ASTER trial found an sICH rate of $6.5 \%$ in the stent retriever group and 5.3\% in the contact aspiration group, similar to our findings with M2 ELVO thrombectomy [8]. Therefore, based on the available data, thrombectomy of M2 ELVO with a stent retriever or a newer-generation pump aspiration catheter may be the optimal choice. This finding will need to be evaluated in a larger randomized trial.

In our multicenter cohort, age was found to be an independent predictor of higher sICH rates, higher mortality rates, and a lower rate of favorable mRS scores in ELVO thrombectomy.

Currently, an increasing number of ELVO patients older than 80 years are receiving mechanical thrombectomy treatment as more safety data are being published. Based on our collaborative data, M2 thrombectomy with a stent retriever or a newer-generation pump aspiration catheter may also prove to be the optimal method compared to manual aspiration. This hypothesis will need to be confirmed in a future study of the elderly AIS thrombectomy population.

The limitations of this study include its retrospective design. The definition of M2 was not prespecified across the different centers. The patient sample dated back to 1999 and only in recent years did newer pump aspiration systems become available. Due to the long duration of the recruitment period, the increased experience of interventionalists in performing thrombectomies could also have affected the results. Other potential limitations from a long recruitment period may include changes in patient selection, thrombectomy techniques, and imaging parameters over time. We also did not utilize central review of angiographic recanalization. Also, the predictive performance of the multivariate analysis model may be overestimated by this analysis because the model has only been tested in the derivation population and not in an independent validation population. However, the strengths of our study include multi-center data, which allowed statistical power to evaluate the difference in outcomes between each thrombectomy technique. Our findings need to be confirmed in a larger, randomized, prospective study utilizing stent retrievers and newer generation pump aspiration systems, because the results would be a significant contribution to finding the optimal technique to treat AIS patients with MCA M2 ELVO.

\section{Statement of Ethics}

The authors confirm that all subjects (or their parents or guardians) have given their written informed consent and that the study protocol was approved by each participating institution's committee on human research. 


\section{Disclosure Statement}

The authors report no disclosures.

\section{Author Contributions}

Study conception and design, data acquisition, and analysis and interpretation of data were done by all of the authors. Drafting of this article was done by Drs. Atchaneeyasakul and Malik. Critical revision of article by Drs. Atchaneeyasakul, Malik, Yavagal, Haussen, Jadhav, Chaturvedi, and Nogueira. Review of the submitted version of this paper was performed by all of the authors.

\section{References}

1 Goyal M, Menon BK, van Zwam WH, Dippel DW, Mitchell PJ, Demchuk AM, et al.; HERMES collaborators. Endovascular thrombectomy after large-vessel ischaemic stroke: a meta-analysis of individual patient data from five randomised trials. Lancet. 2016 Apr;387(10029):1723-31.

2 Powers WJ, Derdeyn CP, Biller J, Coffey CS, Hoh BL, Jauch EC, et al.; American Heart Association Stroke Council. 2015 American Heart Association/American Stroke Association focused update of the 2013 guidelines for the early management of patients with acute ischemic stroke regarding endovascular treatment: A guideline for healthcare professionals from the American Heart Association/American Stroke Association. Stroke. 2015 Oct; 46(10):3020-35.

3 Coutinho JM, Liebeskind DS, Slater LA, Nogueira RG, Baxter BW, Levy EI, et al. Mechanical thrombectomy for isolated $\mathrm{m} 2$ occlusions: A post hoc analysis of the star, swift, and swift prime studies. AJNR Am J Neuroradiol. 2016 Apr;37(4):667-72.

4 Sarraj A, Sangha N, Hussain MS, Wisco D, Vora N, Elijovich L, et al. Endovascular therapy for acute ischemic stroke with occlusion of the middle cerebral artery m2 segment. JAMA Neurol. 2016 Nov;73(11):1291-6.

5 Hacke W, Kaste M, Fieschi C, von Kummer R, Davalos A, Meier D, et al. Randomised double-blind placebocontrolled trial of thrombolytic therapy with intravenous alteplase in acute ischaemic stroke (ECASS II). Second European-Australasian Acute Stroke Study Investigators. Lancet. 1998;352:1245-51.

6 Jankowitz B, Grandhi R, Horev A, Aghaebrahim A, Jadhav A, Linares G, et al. Primary manual aspiration thrombectomy (MAT) for acute ischemic stroke: safety, feasibility and outcomes in 112 consecutive patients. J Neurointerv Surg. 2015 Jan; 7(1):27-31.

7 Kim YW, Son S, Kang DH, Hwang YH, Kim YS. Endovascular thrombectomy for m2 occlusions: comparison between forced arterial suction thrombectomy and stent retriever thrombectomy. J Neurointerv Surg. 2017 Jul; 9(7):626-30.

8 Lapergue B, Blanc R, Gory B, Labreuche J, Duhamel A, Marnat G, et al.; ASTER Trial Investigators. Effect of endovascular contact aspiration vs stent retriever on revascularization in patients with acute ischemic stroke and large vessel occlusion: the aster randomized clinical trial. JAMA. 2017 Aug;318(5):443-52. 